\title{
Systèmes de polyculture élevage laitiers évoluant vers l'AB Renforcement des interfaces cultures/ élevage
}

Evolution towards mixed-crop organic dairy systems : reinforcement of crop/ livestock interfaces

\section{Xavier Coquil, Pascal Béguin et Benoît Dedieu}

\section{OpenEdition}

\section{Journals}

Édition électronique

URL : http://journals.openedition.org/economierurale/4239

DOI : $10.4000 /$ economierurale.4239

ISSN : 2105-2581

\section{Éditeur}

Société Française d'Économie Rurale (SFER)

Édition imprimée

Date de publication : 20 janvier 2014

Pagination : 81-94

ISSN : 0013-0559

Référence électronique

Xavier Coquil, Pascal Béguin et Benoît Dedieu, « Systèmes de polyculture élevage laitiers évoluant vers I'AB Renforcement des interfaces cultures/élevage », Économie rurale [En ligne], 339-340 | janvier-mars 2014, mis en ligne le 20 janvier 2016, consulté le 20 avril 2019. URL : http://journals.openedition.org/ economierurale/4239; DOI : 10.4000/economierurale.4239 


\title{
Systèmes de polyculture élevage laitiers évoluant vers l'AB Renforcement des interfaces cultures/élevage
}

\author{
Xavier COQUIL • Inra UR 055 Aster-Mirecourt, Mirecourt, Inra UMR 1273 Metafort, Saint-Genès- \\ Champanelle \\ coquil@mirecourt.inra.fr \\ Pascal BÉGUIN • Université Lumière Lyon 2, IETL-CMW (UMR 5283), Lyon \\ Benoît DEDIEU • INRA UR 1218, Département SAD, Theix, Saint-Genès-Champanelle
}

Cet article présente, d'un point de vue d'agronome, les connexions entre les cultures et l'élevage dans les systèmes de polyculture élevage ayant évolué vers l'économie en intrants et l'agriculture biologique (AB). II s'appuie sur l'analyse des trajectoires d'évolution (i) d'un système de polyculture-élevage expérimentale de I'INRA, et (ii) de deux exploitations du réseau agriculture durable. L'étude montre que le rapport entretenu par les agriculteurs au territoire de l'exploitation a changé : la recherche d'autonomie et la désintensification s'accompagnent d'une relocalisation de la production agricole et d'une déspécialisation de l'utilisation du territoire de l'exploitation. Les agriculteurs font évoluer leur système vers une nouvelle organisation et un nouveau fonctionnement accentuant les interactions entre cultures et élevage au profit de l'autonomie, ce qui requiert de nouveaux savoir-faire des agriculteurs.

MOTS-CLÉS : polyculture-élevage laitier, autonomie, agriculture biologique, trajectoire

\section{Evolution towards mixed-crop organic dairy systems: reinforcement of crop/livestock interfaces}

In this paper we present, from an agronomist point of view, crop and livestock interactions in mixedcrop dairy systems evolving towards self-sufficient and organic systems. We analyse trajectories of (i) an experimental farm (INRA ASTER-Mirecourt) and (ii) two farms from the RAD network evolving from conventional to self-sufficient and organic systems. Farmers' considerations of the territory change: evolution toward self-sufficiency correspond to less intense use of land, a re-localised agricultural production, and non specialized mobilisation of land. Farmers make their system evolve toward new organisation and operation increasing interactions between crops and livestock that benefit to self-sufficiency. Farmers requiere new know-how to mobilise these interactions. (JEL: Q190).

KEYWORDS: mixed-crop dairy system, self-sufficiency, organic agriculture, trajectories

es systèmes de polyculture élevage
(SPCE) ont un rôle prépondérant à jouer
dans la construction de la sécurité alimen-
taire mondiale (Herrero, Thornton et al.,
2010). Les SPCE sont présentés comme
les archétypes de systèmes permettant des
économies de gamme (Vermersch, 2007)
s'appuyant sur les complémentarités entre
ateliers. Les flux de matières entre élevage
et cultures sont documentés et modélisés
(Nousiainen, Tuori et al., 2011 ; Aubry,
Paillat et al., 2006). En revanche, on connaît moins bien les pratiques des polyculteurséleveurs, à la fois dans le temps et dans l'espace, et la façon dont elles s'ajustent aux variabilités interannuelles. Les registres d'action mis en œuvre par les agriculteurs se limitent-ils à l'organisation de flux entre les ateliers animaux et végétaux?

Notre objectif est ici d'instruire cette question des registres de gestion du fonctionnement des systèmes techniques de 
polyculture élevage sur la base d'études de cas. Nous faisons l'hypothèse que les interactions entre cultures et élevage sont stimulées dans les systèmes autonomes, de façon à limiter le recours aux intrants extérieurs à l'exploitation. Ce travail s'appuie ( $i$ ) sur une étude de trois exploitations de polyculture élevage ayant évolué vers des systèmes en agriculture biologique (AB) et autonomes (recours au minimum d'intrants, et non chimiques) et (ii) sur l'analyse diachronique de leur trajectoire d'évolution en nous centrant sur les interactions entre cultures et élevage. Loin d'une finalité ontologique du fonctionnement des SPCE, l'analyse diachronique vise à identifier $(i)$ des ajustements permis par la combinaison des deux activités, sur des pas de temps inférieur (temps court) ou égal (temps moyen) à une campagne agricole et (ii) ce qui a changé dans les principes de gestion et dans la configuration des systèmes par la mise en place de l'autonomie sur des pas de temps de plus d'une campagne agricole (temps long).

Dans ce texte, nous présentons les trois cas étudiés ainsi que la méthode d'analyse dans une première partie. Nous aborderons ensuite les résultats en portant un éclairage particulier sur les interactions entre cultures et élevage dans les trois systèmes analysés. Les interactions caractérisées entre cultures et élevage sont discutées dans une troisième partie, en focalisant sur les spécificités des systèmes biotechniques en transition vers des systèmes autonomes et biologiques.

\section{Analyse diachronique de trois trajectoires de polyculture élevage évoluant vers l'autonomie}

\section{Matériel : trois exploitations évoluant du conventionnel consommateur d'intrants vers I'AB autonome}

Cette étude est réalisée sur un très petit échantillon, car elle vise à mettre au jour des connexions internes entre cultures et élevage dans les SPCE autonomes et en $\mathrm{AB}$. Les connexions identifiées via l'exploration de ces trois cas seront à confirmer et incrémenter par un élargissement de l'échantillon. Les trajectoires analysées dans le cadre de cette étude proviennent de trois exploitations ayant évolué du conventionnel à l'agriculture biologique et autonome en étant passées par une phase intermédiaire de réduction d'usage d'intrants chimiques. Ces trajectoires proviennent ( $i$ ) d'un système expérimental du dispositif d'expérimentation long terme de l'INRA ASTER-Mirecourt dans la plaine des Vosges, et (ii) de deux exploitations, situées dans le Maine-et-Loire, et adhérentes au Réseau agriculture durable (RAD).

Le système de polyculture élevage du dispositif INRA ASTER-Mirecourt a été étudié sur la période 1985 à 2009 : l'analyse se concentre sur la phase de mise en place du SPCE autonome et biologique à partir de septembre 2004. Cette phase est particulièrement documentée au travers d'une démarche méthodologique de conception/évaluation pas à pas à l'échelle du système de production (Coquil, Fiorelli et al., 2011 ; Coquil, Blouet et al., 2009). Cette démarche de conception/évaluation pas à pas consiste à conduire le système et à le faire évoluer en levant les difficultés techniques qui se présentent et en améliorant son degré d'autonomie. Sur les 240 ha et 100 vaches laitières que totalise le dispositif INRA ASTER-Mirecourt (tableau 1), le SPCE autonome et biologique conçu à partir de septembre 2004 occupe 160 ha (50 ha de prairies permanentes et 110 ha de surfaces cultivées) et comprend 60 vaches laitières (races Holstein et Montbéliarde à parité) et leur renouvellement.

Les deux exploitations du RAD ont été choisies en raison de leur trajectoire d'évolution de systèmes de polyculture élevage conventionnels intensifs à des SPCE biologiques et autonomes. Ces deux exploitations, l'une individuelle et l'autre en 
RECHERCHES

couple, sont de tailles identiques à savoir environ 48 ha de SAU, pour des références laitières situées autour de 210000 litres (tableau 1).

L'essai système de l'INRA ASTERMirecourt et les deux exploitations du RAD se distinguent sur le plan agronomique ainsi que sur le plan du collectif de travail en place. Sur le plan agronomique, une large place est réservée aux cultures de vente dans le SPCE autonome et biologique de l'INRA ASTER-Mirecourt par rapport aux deux exploitations du RAD. L'essai système de l'INRA ASTER-Mirecourt présente un sous-système humain très spécifique puisque composé d'un collectif de recherche en charge de la gestion et de la mise en œuvre concrète de systèmes agricoles expérimentaux. Nous tirons parti de ces spécificités en abordant des registres essentiellement d'ordre technique, à partir des données de Mirecourt : la précision des données disponibles permet d'analyser les ajustements sur le temps moyen et court. Les registres d'ordre humain sont traités à partir des données des enquêtes dans les exploitations du RAD : ces deux fermes, proche sur le plan biotechnique, offrent des collectifs de travail très distincts (individuel versus couple).

\section{Méthode : recueil et analyse diachronique des trajectoires d'exploitations}

Ce travail a été conduit selon des méthodes de recueil et d'analyse de données mobilisées dans des travaux relevant d'approches long terme de l'exploitation agricole (Levrouw, Morales et al., 2007 ; Begon, Pailleux et al., 2009 ; Cialdella et Dedieu, 2010).

Tableau 1. Caractéristiques biotechniques principales des systèmes de polyculture élevage étudiés au cours de leur trajectoire

\begin{tabular}{|c|c|c|c|c|c|c|}
\hline \multirow{2}{*}{$\begin{array}{c}\text { Transition } \\
\text { Exploitations }\end{array}$} & \multicolumn{3}{|c|}{ Phase : SPCE conventionnel et intensif } & \multicolumn{3}{|c|}{ Phase : SPCE AB et autonome } \\
\hline & $\begin{array}{l}\text { INRA ASTER- } \\
\text { Mirecourt }\end{array}$ & RAD1 & RAD2 & $\begin{array}{l}\text { INRA ASTER- } \\
\text { Mirecourt }\end{array}$ & RAD1 & RAD2 \\
\hline SAU & 200 & 24 & 30 & 160 & 47 & 49 \\
\hline $\begin{array}{l}\text { Prairies naturelles } \\
\text { (ha) }\end{array}$ & 140 & 1.5 & 0 & 50 & 3 & 0 \\
\hline $\begin{array}{l}\text { Prairies tempo- } \\
\text { raires (ha) }\end{array}$ & 0 & 10 & 10 & $\begin{array}{c}50 \text { (dépend } \\
\text { de la rotation) }\end{array}$ & 38 & 44 \\
\hline Céréales (ha) & $\begin{array}{l}30 \text { (variation } \\
\text { selon surface } \\
\text { de maïs) }\end{array}$ & 3 & 10 & $\begin{array}{c}50 \\
\text { (dépend de la } \\
\text { rotation) }\end{array}$ & 0 & 0 \\
\hline $\begin{array}{l}\text { Associations } \\
\text { céréales/protéagi- } \\
\text { neux (ha) }\end{array}$ & 0 & 0 & 0 & $\begin{array}{l}10 \text { (dépend de } \\
\text { la rotation) }\end{array}$ & 3 & 3 \\
\hline Maïs (ha) & 30 (maximum) & 10 & 10 & 0 & 3 & 2 \\
\hline \multicolumn{7}{|l|}{ Troupeau } \\
\hline $\begin{array}{l}\text { Effectifs vaches } \\
\text { laitières }\end{array}$ & 140 à 150 & 30 & 20 & 60 & 42 & 35 \\
\hline Race & $\begin{array}{l}\text { Holstein et } \\
\text { Montbéliarde }\end{array}$ & Holstein & Holstein & $\begin{array}{l}\text { Holstein et } \\
\text { Montbéliarde }\end{array}$ & $\begin{array}{l}\text { Holstein/ } \\
\text { Montbé- } \\
\text { liarde/ } \\
\text { croisée }\end{array}$ & Holstein \\
\hline $\begin{array}{l}\text { Élevage des } \\
\text { génisses }\end{array}$ & Toutes & $\begin{array}{c}\text { Renouvel- } \\
\text { lement }\end{array}$ & $\begin{array}{c}\text { Renouvel- } \\
\text { lement }\end{array}$ & Toutes & $\begin{array}{c}\text { Renouvelle- } \\
\text { ment }\end{array}$ & $\begin{array}{c}\text { Renouvel- } \\
\text { lement }\end{array}$ \\
\hline Élevage des mâles & Tous & Non & Non & Non & Non & Non \\
\hline
\end{tabular}


Les trajectoires ont été recueillies en mai 2009 dans le cadre d'entretiens semidirectifs d'environ deux heures, pour les exploitations du RAD, et trois entretiens de deux heures pour le SPCE ASTERMirecourt. Ces entretiens visaient à cerner les phases traversées par l'exploitant, de son installation à aujourd'hui, en décrivant les configurations et les fonctionnements de leur exploitation pour chaque phase. Concernant le SPCE ASTER-Mirecourt, cette description est complétée $(i)$ par des données biotechniques issues de l'évaluation agronomique permettant le suivi de l'élaboration des performances techniques du système, et (ii) par la connaissance, de par l'implication de l'un des auteurs, des conduites en place sur le dispositif.

L'analyse a permis de mettre en évidence trois phases pour chacun des systèmes de l'installation à l'enquête : agriculture conventionnelle usant d'intrants chimiques de manière intensive, agriculture conventionnelle plus autonome en intrants et enfin agriculture biologique autonome en intrants. L'analyse porte essentiellement sur le passage des exploitations à des systèmes autonomes en $\mathrm{AB}$. Les variations et invariants des systèmes tant sur le plan de leur configuration que sur le plan de leur fonctionnement sur des pas de temps longs, moyens ou courts ont été analysés. Ainsi, le passage à l'autonomie nous sert de « révélateur » d'une partie des interactions entre cultures et élevage.

\section{Résultats : Systèmes $A B$ autonomes Renforcement aux interfaces entre cultures et élevage}

Dix registres différents rendent compte des variations et des invariants entre les trois phases des trois trajectoires étudiées et des ajustements intra-phases. Ces registres d'analyse mettent en évidence les variations et les invariants de configuration et de fonctionnement des systèmes de polyculture élevage étudiés au cours de leur trajectoire. Nous référant à la représentation des systèmes agricoles selon deux sous-systèmes, l'un biotechnique et l'autre humain (Osty et Landais, 1993) (i) quatre registres caractérisent des éléments relatifs au sous-système biotechnique : le territoire, le couple troupeau/ressource, les cultures et les infrastructures (bâtiment, matériel...) (ii) cinq registres caractérisent les éléments relatifs au sous-système humain : le travail, la formation, les centres d'intérêt, les valeurs et le projet (iii) un registre caractérise la mise en mouvement des deux sous-systèmes dans le temps.

L'évolution vers des SPCE autonomes et en $\mathrm{AB}$ renforce les interfaces entre cultures et élevage. Les systèmes de polyculture élevage autonomes et en $\mathrm{AB}$ fonctionnent, quasi exclusivement, à partir des ressources internes, c'est-à-dire des ressources présentes sur l'exploitation. Dans ce chapitre, nous présentons les conséquences de ce nouveau fonctionnement : une relocalisation de l'activité sur la ferme, une déspécialisation de l'usage des sols du parcellaire, la mise en place de nouveaux ajustements entre cultures et élevage permettant de gérer les variations de disponibilités des ressources internes et la mise en place de nouvelles pratiques de gestion impliquant les interfaces cultures/ élevage. Nous abordons la diversité des attentes des agriculteurs vis-à-vis des cultures annuelles et fourragères dans ces systèmes.

\section{Le passage à l'autonomie Relocalisation de l'activité et déspécialisation du territoire}

La mise en place de systèmes autonomes et biologiques s'est traduite dans les trois systèmes étudiés par (i) une diminution de la productivité agricole par hectare (ii) une diminution de l'utilisation de facteurs de production externes (arrêt de l'usage d'intrants de synthèse, arrêt d'achat de 
fourrages...), (iii) la sous-réalisation de la référence laitière et (iv) la diminution (SPCE ASTER-Mirecourt), voire l'arrêt (RAD1 et RAD2) de la production de cultures de vente. Pour le SPCE ASTERMirecourt, l'atelier de bœufs et de taurillons laitiers a été supprimé.

L'évolution de ces trois systèmes sur le temps long témoigne d'un changement de rapport des agriculteurs au territoire : le passage à l'autonomie correspond à une relocalisation de la production agricole et une déspécialisation des secteurs du territoire de l'exploitation.

La relocalisation s'exprime par un changement de logiques d'assolement et la distinction de potentialités agronomiques à la parcelle. Ainsi, dans le SPCE ASTERMirecourt, les trois logiques d'assolement qui se sont succédé durant les trois phases de la trajectoire sont : un assolement basé sur la recherche de la couverture des besoins annuels en fourrage du troupeau, un autre pour couvrir les besoins du troupeau et les obligations légales (jachère...) puis, dans le système autonome et biologique, un dernier basé sur (i) les potentialités du milieu (figure 1), (ii) les fonctionnalités du parcellaire, (iii) l'autonomie alimentaire (fourrages et concentrés) ainsi qu'en paille du troupeau. Dans les deux autres exploitations, la logique d'assolement était calée sur des besoins fourragers puis, depuis le passage à l'autonomie, sur la volonté d'être autonome en fourrages, en concentrés et sur la prise en compte des potentialités de chaque parcelle pour le choix des espèces, et des variétés cultivées.

La déspécialisation du territoire agricole s'exprime par l'introduction des cultures annuelles sur les îlots de prairie précédemment réservés au troupeau, mais aussi par l'introduction de prairies dans les blocs de parcelles précédemment réservés aux cultures. Pour Mirecourt, qui pratique le pâturage de longue date, les cultures annuelles (céréales) ont fait leur apparition dans les îlots de pâturage des vaches laitières. Pour les deux exploitations du RAD - qui sont passées du 0 pâturage à un pâturage maximisé -, les vaches laitières ont été introduites dans le parcellaire anciennement cultivé.

Dans la ferme INRA, la mise en place de systèmes autonomes est l'objet de l'activité de conception innovante de systèmes techniques (Coquil, Blouet et al., 2009). Elle s'inscrit dans un dialogue entre

Figure 1. Configuration du territoire de l'installation expérimentale de l'INRA de Mirecourt (240 ha) à partir de la caractérisation, à dire d'experts, des potentialités agricoles des parcelles.

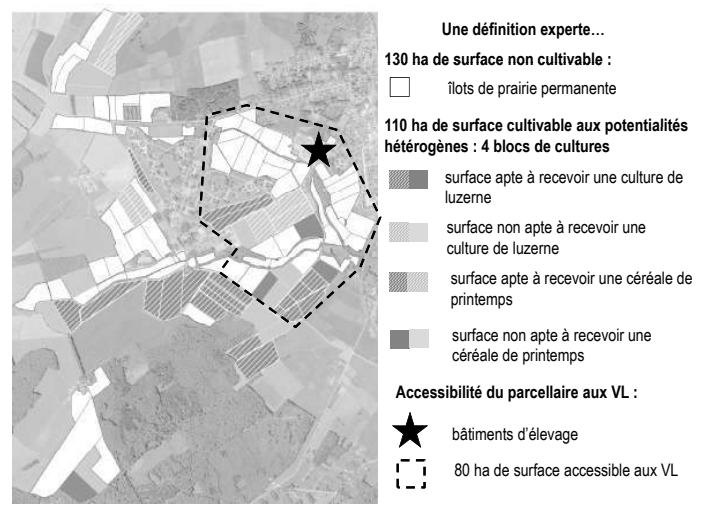


chercheurs de disciplines différentes et techniciens, également dans une évolution des compétences des agents expérimentaux. Dans les deux exploitations du RAD, les changements de modes de gestion et d'entités gérées font l'objet d'acquisition de nouveaux savoir-faire stimulés par des dynamiques de groupes (échanges entre pairs et formations). Mais le passage à des systèmes autonomes et biologiques dans ces deux exploitations dépasse ces changements progressifs d'ordres techniques. Le projet d'autonomie dans ces deux exploitations correspond aussi à un projet éthique, une recherche de cohérence entre des idées (autonomie, économie en intrants, indépendance vis-à-vis des négociants...) et des pratiques agricoles, commerciales... Cette recherche de cohérence se traduit sur le plan des pratiques «être en phase avec ses idées » : dans les deux exploitations enquêtées au RAD, les investissements sont gérés de manière très économe, l'un des deux exploitants se lance dans la transformation et la commercialisation de ses produits alors que l'autre cherche un mode de transmission de son exploitation en phase avec ses idées afin de préparer son départ à la retraite. Ainsi, pour ces deux exploitations agricoles, le projet de systèmes autonomes et biologiques correspond également à une stimulation intellectuelle perpétuelle et à la mise en place de projets agricoles sans envisager l'agrandissement de l'exploitation.

Les systèmes de polyculture élevage autonomes et biologiques présentés dans ce travail représentent des projets d'agriculture située, car basée sur la mobilisation, la plus exclusive possible, des ressources locales au sein des territoires. Dans les trois exploitations suivies, la mise en place de la conduite ajustée, afin de faire avec les ressources disponibles au sein des systèmes, nécessite d'abandonner le projet d'expression maximale de la performance technique des animaux et des cultures. L'activité agricole change de nature : "On laboure moins et on observe plus afin d'anticiper les évolutions... »(RAD2).

\section{Apparition d'interfaces d'intérêts techniques entre cultures et élevage}

Sur le dispositif expérimental de Mirecourt, le passage à une forme d'agriculture plus autonome a débuté en 1985, mais le passage à l'agriculture autonome et biologique, que nous instruisons plus particulièrement dans cette partie, a démarré en septembre 2004. La mise en œuvre du projet de polyculture élevage autonome et biologique s'est traduite par un apprentissage progressif de l'usage des systèmes d'élevage dans les systèmes de cultures et réciproquement.

Sur le plan animal, ceci se traduit par l'apprentissage progressif de l'utilisation de ressources variées pour nourrir le troupeau. En effet, l'autonomie a consisté à nourrir le troupeau avec les ressources dont on dispose sur le territoire en (i) mettant en place une gestion des surfaces fourragères et cultivées afin d'obtenir des ressources fourragères d'intérêt zootechnique et (ii) palliant les variations de disponibilités des ressources (tableau 2) en raison des variations annuelles des soles fourragères, des soles cultivées et de leurs rendements.

- À compter de la campagne 2007, la mise à l'herbe des génisses du SPCE ASTERMirecourt a été réalisée sur les prairies temporaires alors qu'elle avait lieu sur des prairies permanentes depuis 1972 : ce changement visait à réaliser un déprimage de ces nouveaux couverts, afin de différer leur fauche de fin mai/début juin à juin et ainsi assurer une meilleure probabilité de récolte sous forme de foin et non d'enrubannage. Cet exemple de gestion combinée du pâturage et de l'élaboration des stocks a permis d'augmenter la proportion de fourrages conservés sous forme sèche à compter de 2007 (tableau 2). 
RECHERCHES

Xavier COQUIL, Pascal BÉGUIN, Benoit DEDIEU

Tableau 2. Ressources récoltées, hors pâturage, pour l'alimentation, la litière et la complémentation du troupeau SPCE de l'INRA ASTER-Mirecourt

\begin{tabular}{|c|c|c|c|c|}
\hline Modalités récolte & Ressources (tMS/an) & 2006 & 2007 & 2008 \\
\hline Enrubannage-ensilage d'herbe & - & 41 & 32 & 38 \\
\hline \multirow[t]{3}{*}{ Foin } & Luzerne/dactyle & 118 & 135 & 133 \\
\hline & Prairie temporaire & 9 & 81 & 53 \\
\hline & Prairie permanente & 89 & 107 & 103 \\
\hline Total & Fourrages & 256 & 355 & 326 \\
\hline \multirow[t]{3}{*}{ Total } & Paille & 144 & 85 & 152 \\
\hline & $\begin{array}{l}\text { Mélanges céréales/ } \\
\text { prot1 }\end{array}$ & 46,0 & 15,1 & 7,0 \\
\hline & Céréale secondaires 2 & 46,8 & 34,2 & 38,3 \\
\hline Total & Concentrés & 92,9 & 49,3 & 45,3 \\
\hline
\end{tabular}

Notes : 1 : avoine/féverole ; triticale/pois ; orge/lupin... ; 2 : orge, triticale, avoine, épeautre, seigle... ;

MS : matière sèche.

Source : Trommenschlager, JM., Fiorelli, JL, Coquil, X..

- Les logiques d'assolement, de conduite des cultures fourragères et d'achat d'aliments en système conventionnel intensif permettaient d'assurer des rations alimentaires assez constantes et équilibrées sur le plan énergétique et azoté durant l'année. Après conversion, dans une logique d'autonomie, l'apprentissage de l'ajustement du troupeau aux ressources à différents pas de temps a été nécessaire afin de faire face aux variations de disponibilité des ressources durant le pâturage ainsi que durant la période d'affouragement hivernale. Le pâturage des vaches laitières du SPCEASTER-Mirecourt peut être constitué de prairies permanentes, de prairies temporaires et de luzerne/dactyle : la gestion combinée des stocks et du pâturage ainsi que les difficultés pratiques à faire pâturer des luzernes par des vaches laitières nous invitent à mobiliser le pâturage luzerne/dactyle uniquement en période de manque de fourrages, la luzerne présentant une résistance intéressante au stress hydrique (figure 2). Des ajustements sur le moyen et court terme ont lieu lors de l'affectation des ressources stockées pour l'élaboration des rations hivernales : ainsi les veaux et les vaches laitières sont les premiers animaux servis, comme en atteste la relative constance de leur ration d'une année à l'autre (tableau 3), et les génisses en croissance sont alimentées par les stocks restants palliant les variations de disponibilité des stocks (tableau 3). Des ajustements sur le plus long terme par la réforme anticipée ou la vente d'animaux sont également intervenus avant l'hiver 2007 pour faire face à des stocks en fourrages et en paille trop faibles pour passer l'hiver (vente de 8 vaches laitières (dont 4 réformes anticipées) et 5 génisses de 24 mois).

Sur le plan des systèmes de culture, l'introduction de la prairie temporaire, et par la même occasion de l'animal pâturant, dans le territoire auparavant uniquement destiné aux cultures annuelles, a permis aux animaux d'avoir des actions directes et indirectes dans les systèmes de cultures.

- Ainsi, le pâturage des animaux à faibles besoins de croissance, telles que les génisses en fin de gestation, a permis de limiter le salissement des parcelles de prairies temporaires en rumex et ainsi de limiter le salissement à l'échelle de la rotation. La présence d'animaux dans 
Les transversalités de l'agriculture biologique

Figure 2. Diversité des prairies pâturées par les vaches laitières du SPCE au cours de la saison 2008

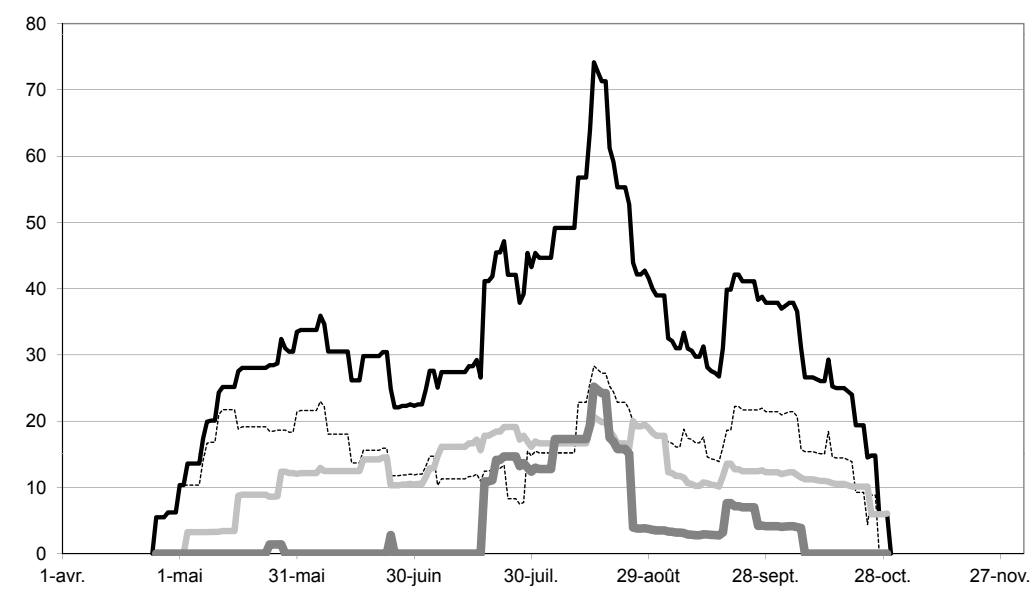

Source : les auteurs.

Tableau 3. Variations interannuelles des régimes alimentaires, hors pâturage, des lots d'animaux du SPCE

\begin{tabular}{|c|c|c|c|c|c|c|c|}
\hline \multirow{2}{*}{$\begin{array}{l}\text { Modalités } \\
\text { de récolte }\end{array}$} & \multirow{2}{*}{$\begin{array}{l}\text { Ingestion } \\
\text { des ressources } \\
\text { (kg MS/VL/an) }\end{array}$} & \multicolumn{3}{|c|}{$2006 / 2007$} & \multicolumn{3}{|c|}{$2007 / 2008$} \\
\hline & & VL & $\begin{array}{l}\text { Veaux } \\
\text { (0-1 an) }\end{array}$ & $\begin{array}{l}\text { Génisses } \\
\text { (1 an-vêlage) }\end{array}$ & VL & $\begin{array}{l}\text { Veaux } \\
\text { (0-1 an) }\end{array}$ & $\begin{array}{c}\text { Génisses } \\
\text { (1 an-vêlage) }\end{array}$ \\
\hline $\begin{array}{l}\text { Ensilage- } \\
\text { enrubannage }\end{array}$ & & 26 & 0 & 1235 & 270 & 0 & 71 \\
\hline \multirow[t]{3}{*}{ Foin } & Luzerne-dactyle & 1177 & 394 & 10 & 1320 & 244 & 272 \\
\hline & Prairie temporaire & & 0 & 0 & 590 & 0 & 965 \\
\hline & Prairie permanente & 1390 & 73 & 985 & 700 & 14 & 723 \\
\hline Paille & & 0 & 0 & 127 & 0 & 0 & 0 \\
\hline Refus & & 0 & 0 & 0 & 0 & 0 & 346 \\
\hline \multirow[t]{3}{*}{ Total } & Fourrages & 2601 & 469 & 2358 & 2880 & 258 & 2377 \\
\hline & Mélanges céréales/prot ${ }^{1}$ & 509 & 112 & 2 & 198 & 67 & 2 \\
\hline & Céréales $^{2}$ & 72 & 66 & 42 & 421 & 77 & 11 \\
\hline Total & Concentrés & 586 & 179 & 44 & 652 & 144 & 13 \\
\hline
\end{tabular}

Notes : 1 : avoine/févérole ; triticale/pois ; orge/lupin... ; 2 : orge, triticale, avoine, épeautre, seigle... ;

MS : matière sèche.

Source : les auteurs.

le système a permis la valorisation $(i)$ de prairies temporaires aux fonctions agronomiques intéressantes à l'échelle de la rotation culturale : fixation azotée, apports de matières organiques au sol, rupture des cycles d'adventices annuelles... (ii) de valoriser, sous forme de fourrages récoltés, des essais de grandes cultures manqués...

- La présence d'une tête de rotation pluriannuelle en fourrages a fait apparaître le pas de temps de la rotation dans la 
gestion du travail du sol (plus on s'approche de la tête de rotation et plus on autorise la présence d'adventices annuelles comptant sur l'effet nettoyant de la tête de rotation fourragère) et de la fertilisation (le retournement de prairies entraîne une libération d'azote).

L'émergence de ces nouvelles fonctions entre cultures et élevage par la situation d'autonomie a entraîné, certes, de nouvelles interactions, mais aussi de nouvelles interdépendances entre cultures et élevage et ainsi de nouvelles contraintes notamment calendaires et fonctionnelles.

La recherche d'un équilibre entre production de ressources et utilisations internes potentielles est à la fois faite sur les pas de temps annuel et pluriannuel (démographie de troupeau, reports de stocks...), mais également au fur et à mesure du déroulement du calendrier. Du coup, ces équilibres entre cultures et élevage sont très dynamiques et soumis aux aléas notamment climatiques : ainsi, le passage à l'autonomie a placé le collectif de Mirecourt dans l'apprentissage d'une gestion adaptative, quittant progressivement l'optimisation et la sécurisation de la performance animale et végétale par la mobilisation d'intrants, et requérant de nouvelles pratiques et indicateurs d'observations de l'état du système.

- Le passage à un système biologique et autonome demande de pallier l'absence de sécurisation par les intrants. Ceci a nécessité la mise en place : (i) de nouveaux leviers de gestion et (ii) de pratiques agricoles plus préventives, en réponse à des principes de précautions. Par exemple, en système conventionnel, l'équilibre alimentaire était géré par des achats d'aliments ou un ajustement de la fertilisation azotée des prairies au printemps, voire un ajustement de la sole de maïs d'une année à la suivante : ces leviers d'ajustements de la production fourragère ont été supprimés lors de la conversion à l'AB autonome, faisant apparaître l'ajustement par une gestion des effectifs et de la démographie du troupeau. L'arrêt d'utilisation des désherbants au sein des systèmes de culture a été anticipé par la mise en place de rotations culturales conçues afin de rompre les cycles des adventices par des alternances de cultures annuelles et pluriannuelles, et par des alternances de cultures de printemps et de cultures d'hiver lorsque la portance des parcelles au printemps le permettait.

- Ces nouvelles interactions et interdépendances entre cultures et élevage nécessitent d'identifier et de suivre l'état des ressources disponibles afin d'ajuster les conduites selon leur degré de disponibilité. Ainsi, le passage à un système autonome et biologique a engagé le collectif de l'installation de Mirecourt dans un apprentissage progressif à de nouvelles observations, car les ressources mobilisées sont en perpétuelles évolutions en fonction, notamment, des usages qui en sont faits.

\section{Le rôle central de la culture de l'herbe}

Chez RAD1 et RAD2, la mise en place de systèmes de polyculture élevage autonomes et biologiques s'est traduite par un recentrage sur la culture de l'herbe. Durant leur période conventionnelle, les cultures annuelles étaient destinées à la vente, ce qui entrainait une forte pression sur les surfaces fourragères pour nourrir le troupeau. Le passage à des systèmes autonomes et biologiques a été conduit par un recentrage sur l'activité laitière et la conception progressive d'un système alimentaire autonome pour les vaches : concrètement, l'autonomie s'est traduite par la mise en culture et la valorisation des surfaces en herbe au détriment du maïs, lequel nécessitait, en outre, une complémentation en correcteur azoté importé. 
Une diversité de ressources alimentaires est mise à disposition du troupeau, avec des usages plus ou moins spécifiés (encadré 1), par le biais de modes d'exploitation différenciés de l'herbe, de choix de variétés implantées et d'espèces variées. L'apprentissage de la gestion combinée du pâturage et de l'élaboration des stocks semble avoir un caractère central dans ces deux systèmes autonomes. Chez RAD1 et sur le SPCE ASTER-Mirecourt, la gestion de la qualité de l'herbe pâturée est passée par la mise en place d'un lot d'animaux spécifique (bovins suiveurs avec des animaux à faibles besoins alimentaires : génisses âgées, vaches taries...), pâturant les refus des parcelles pâturées par les vaches laitières afin d'assurer des repousses de qualité. Chez RAD1 et sur le SPCE ASTER-Mirecourt, la diversité des ressources fourragères stockées est mise à profit de la confection des rations hivernales en hiérarchisant l'attribution des ressources aux lots selon des logiques proches (encadré 1 et tableau 3)
L'apprentissage de la culture de l'herbe a un caractère central dans la conduite de ces systèmes. Chez RAD1 et RAD2, la faible proportion de cultures annuelles (céréales essentiellement) présente dans ces systèmes autonomes et biologiques trouve, à l'origine, sa justification essentielle dans la volonté de renouveler les prairies afin de stimuler leur productivité : pour ces personnes, la réimplantation d'une prairie est facilitée lors de la mise en culture de la parcelle durant au moins une année. Toutefois, les cultures annuelles présentes dans la rotation ont été mises au service de l'autonomie alimentaire du troupeau (i) créant progressivement de nouvelles interactions entre cultures et élevage, (ii) facilitant l'utilisation de cultures annuelles telles que les céréales sous forme de fourrages en cas de salissement de la culture. Ainsi, la culture d'une petite sole de maiis dans ces deux systèmes est devenue intéressante en vue d'apporter un complément fourrager énergétique permettant une meilleure valorisation de l'herbe de printemps

\section{Encadré 1. Témoignage d'un éleveur du RAD sur la réalisation d'une diversité de fourrages stockés à destination de l'alimentation hivernale}

«Ça, c'est l'approche pâturage, on est dans du multi-espèces surtout, c'est un peu plus compliqué $[. .$.$] on a eu de l'eau et un peu de cha-$ leur depuis un mois c'est assez dense et donc le multi-espèce est un peu plus dur à pâturer, elles [les VL] laissent un peu plus de refus : je me retrouve avec du dactyle, fétuque et trèfle violet qui sont montés un peu plus vite que prévu. Donc, j'ai mis en fauche 2 ha... euh, 3 ha hier que j'avais pas prévu. [...] il y a une parcelle en dominance dactyle qui passe bien au déprimage mais qui ensuite est dure à faire pâturer en mai et plus loin j'ai 2 ha en dominance fléole que je laisse également en fauche et la fléole c'est une plante très intéressante en foin : c'est une plante appétante et à mon avis très digeste [...] en plus il $\mathrm{y}$ a beaucoup de trèfle blanc dedans donc ça me permet d'avoir un foin intéressant pendant l'hiver [...]. Je fais différents foins : ce que j'ai fauché hier par exemple, c'est ce qui va me servir de foin de production, c'est de l'herbe tendre, pas mature, qui servira de fourrage de production et va rester en foin plus mécanique, plus de santé animale, les fléoles, dactyles... qui seront fauchés mi-juin à maturité presque en graines et la luzerne est un foin de tampon dans la panse. Ensuite j'ai des foins de prés à flore très variée : c'est une autre catégorie. Dans mon hangar tout est classé : les bottes sont par colonnes avec foin de production, foin mécanique, foin de prés, foins de luzerne et on peut avoir des foins de flore intermédiaire. [...] En fonction de ce que les vaches expriment en termes de poils, en termes de bouses, on change... on a toujours une période de tâtonnement en automne... c'est une contrainte de faire 5 foins...là j'ai 5 ha que je fais attendre [...]. » 
par les vaches pour RAD 1 et d'intensifier la production de fourrages et la production laitière afin de remonter ponctuellement la trésorerie pour RAD2.

Dans ces deux exploitations du RAD, le passage à des systèmes autonomes et biologiques a nécessité la mise en place de conduites ajustées, essentiellement sur le plan animal. Ce passage a également nécessité un apprentissage renouvellé des observations menées sur les entités gérées en perpétuelle évolution, en mobilisant également les observations d'intervenants extérieurs sur l'exploitation «je compte sur le coup d'oeil de l'inséminateur pour ajuster la ration » (RAD 1). Dans ces deux exploitations, l'autonomie en matière est un principe sur le temps long ( $i$ ) pouvant faire l'objet d'aménagements pour pallier des déficits ponctuels de fourrages (achats ponctuels de luzerne déshydratée...), (ii) pouvant être élargi à une échelle dépassant le territoire de l'exploitation agricole en intégrant le réseau de travail de l'agriculteur afin d'organiser des échanges (exemple : échanges de tourteaux de colza contre du fumier) : ainsi des leviers de flexibilité apparaissent notamment en transigeant au principe d'autonomie de matière.

\section{SPCE vers I'AB autonome Interdépendance des cultures et de l'élevage ?}

L'évolution de ces trois systèmes de polyculture élevage vers une agriculture biologique et autonome marque des changements de fonctionnement des systèmes de production au profit d'interactions renforcées entre cultures et élevage. Ces interactions accentuent la connectivité entre éléments internes du système (Holling, 2001), notamment entre le troupeau et les couverts, rendant leur interdépendance plus forte et, ainsi, nécessitant parfois de rendre compatible leurs inscriptions temporelles par des modifications de pratiques. C'est, par exemple, le cas dans le cadre des modifications de conduite du pâturage des génisses sur le SPCE ASTER-Mirecourt afin de concilier pâturage et récoltes de prairies temporaires sous forme de foins.

\section{Des évolutions biotechniques spécifiques des transitions}

La transition de systèmes agricoles à des situations autonomes et biologiques impose l'apparition de processus biotechniques spécifiques tels que l'adaptation du troupeau à de nouvelles ressources fourragères présentant de plus fortes variations de qualité et de quantité intra et inter-campagnes (Gouttenoire, Fiorelli et al., 2010), l'adaptation des sols et des systèmes de culture à une fumure en azote plus modérée et plus lente, l'adaptation des conduites des systèmes de culture à de forts salissements adventices. Dans une synthèse internationale, Lamine et Bellon (2009) font état du peu d'études portant sur les états intermédiaires des systèmes biotechniques durant la conversion des exploitations agricoles du conventionnel à l'agriculture biologique. Les modifications de quelques entités biotechniques ont été abordées telles que (i) le fonctionnement biologique et, par conséquent, les fonctionnements physiques (structure) et chimiques (cycles des éléments) des sols (Lotter, 2003), et (ii) les modifications des rotations culturales et l'importance de « l'effet précédent» pour faire face à une disponibilité limitée des éléments minéraux dans les sols. À ces études agronomiques, nous ajoutons la contribution des travaux issus de l'écologie des perturbations. Albrecht (2005) s'intéressant à l'évolution de la flore adventice dans les parcelles cultivées lors de la conversion à l'agriculture biologique, fait état d'une période d'instabilité de cette flore durant environ six ans à partir de la modification des pratiques culturales.

\section{Transition vers l'autonomie Vers des systèmes autonomes résilients ?}


Les connexions entre cultures et élevage permettent de limiter le recours aux intrants d'origines fossiles ou chimiques (engrais, pesticides...) ou autres (fourrages, concentrés, paille...) grâce à la création de ressources productives dans le système (nouvelles cultures faisant office de concentrés, besoins en concentrés qui évoluent grâce à une production fourragère ayant changé de valeur alimentaire [équilibre entre énergie et azote]...).

L'augmentation de la connectivité entre production de ressources et utilisations internes a entraîné l'apparition de nouvelles pratiques d'ajustement (effectifs animaux, cultures à usages multiples, élargissement des frontières du système par des échanges avec les voisins...) et la mise en place d'entités biotechniques à usages multiples (fourrages pâturables et fauchables, cultures vendables ou mobilisables pour les animaux, des animaux aux fonctionnalités agronomiques directes ou indirectes multiples...). Ainsi, ces nouvelles ressources peuvent jouer un rôle dans la sécurisation des systèmes face aux aléas.

L'augmentation de la connectivité entre ressources internes au système ne semble donc pas nécessairement aller de pair avec une spécialisation des ressources via leur usage tel que suggéré par Holling (2001). Dans sa conceptualisation des systèmes socio-écologiques selon un cycle inscrit dans un plan en trois dimensions, Holling (2001) les présente allant vers l'autonomie comme des systèmes au sein desquels la connectivité augmente entre les éléments internes, ayant pour conséquence de réduire leur résilience. Ainsi, selon Holling (2001), cette forte connectivité conduit à une fragilité du système qui sera contraint à être profondément reconçu lors de la venue d'un choc empêchant certaines interdépendances de se réaliser. Toutefois, Holling (2001) précise que ces situations présentent un fort potentiel de reconception.

\section{Interactions cultures/élevage Poids relatifs des cultures et de l'élevage ?}

Le passage à un système autonome et biologique a marqué pour les trois systèmes un changement de production agricole. Le SPCE ASTER-Mirecourt vend du lait, du blé meunier et des vaches laitières réformées alors qu'il commercialisait auparavant plus de lait, du blé à destination de l'alimentation animale, du colza, des vaches de réformes ainsi que l'ensemble des mâles nés qui étaient engraissés et vendus en boeufs de 30 à 36 mois ou en taurillons de 18 à 24 mois. Les deux exploitations du RAD ont arrêté la vente de cultures (blé, production de semences fourragères) et ont axé la commercialisation sur la vente de lait.

Ainsi se dégagent deux projets à partir de ces trois cas de polyculture élevage laitier autonomes influençant fortement la configuration et le fonctionnement des systèmes. Un projet de commercialisation centré sur le lait produit à l'herbe, et un projet de commercialisation de lait et de blé dans un système autonome en aliments (fourrages, concentrés) et en litière (paille).

- Ces projets correspondent à deux configurations du territoire de l'exploitation agricole : l'une basée sur la mise en culture de l'herbe et l'autre sur la mise en place d'un équilibre entre production fourragère et production de cultures. Ainsi, l'implantation de la prairie temporaire est réalisée suivant des objectifs agronomiques dans les systèmes de culture du SPCE ASTER-Mirecourt (casser le cycle des adventices, apporter de l'azote et de la matière organique) et dans les deux exploitations enquêtées du RAD ce sont les cultures annuelles qui sont implantées suivant des objectifs agronomiques (permettre le renouvellement des prairies). 
- À partir d'un socle commun de polyculture élevage autonome et biologique, les attentes des agriculteurs vis-à-vis des cultures annuelles et fourragères dans leur rotation sont très variables : les interactions fonctionnelles en place entre cultures et élevage sont alors très différentes. Toutefois, dans un processus de transition vers des systèmes autonomes et biologiques, ces attentes vis-à-vis des ressources peuvent aussi être évolutives : les deux agriculteurs du RAD reconnaissent, via leur expérience progressivement acquise par l'usage de cultures annuelles dans leur système d'élevage, des intérêts zootechniques à ces cultures. Ainsi, RAD1 est attiré par la présence d'un fourrage énergétique tel que le maïs pour complémenter l'herbe pâturée de printemps, ce qui l'incite aujourd'hui à tester une culture annuelle alternative : la betterave. RAD2 reconnaît des apports minéraux intéressants par l'épeautre dans les rations des veaux l'incitant à systématiser cette culture dans sa rotation...

\section{Transitions des SPCE vers l'autonomie Émergence de nouvelles connexions internes au système}

Une première analyse diachronique des trajectoires de mise en place de ces trois systèmes de polyculture élevage autonome a permis de mettre en exergue une configuration des systèmes de production qui devient localisée, c'est-à-dire à partir des potentialités agronomiques disponibles sur l'exploitation. La mise en place de ce principe de configuration à partir des potentialités agronomiques se traduit par la mise en place de modalités de conduites adaptatives des systèmes afin de pallier les variations de productivité de ce territoire aux dimensions finies. Ces conduites adaptatives mobilisent les interactions possibles entre ressources internes du système. Dans le cadre des systèmes de polyculture élevage, les animaux et les cultures sont des ressources et des utilisateurs potentiels et mutuels : l'analyse diachronique des trajectoires de mise en place de ces systèmes met en exergue l'apparition progressive de ces liens fonctionnels entre ces entités et les modifications systémiques qu'elles génèrent. L'utilisation des génisses pour pâturer et retarder les dates de fauche, puis la découverte de leur capacité à pâturer des rumex et ensuite leur utilisation pour limiter le «salissement rumex » des parcelles de prairies temporaires dans les rotations culturales... Ces transitions entraînent l'émergence de nouvelles propriétés biotechniques (des vaches adaptées à des fluctuations alimentaires...), de nouvelles règles de conduite (apprendre le pâturage et la culture de l'herbe, ajuster les rations selon les disponibilités alimentaires...) qui nécessitent l'acquisition de nouveaux savoir-faire pour les agriculteurs qui décident, pilotent et agissent : quels sont les processus, les ressources et les situations impliqués dans cette acquisition de nouveaux savoir-faire durant la transition vers des systèmes plus autonomes?

Les auteurs remercient le collectif de l'unité de recherche INRA ASTER-Mirecourt et le Réseau Agriculture Durable pour leurs contributions à ce travail. Ce travail a bénéficié du soutien financier du programme ANR Systerra O2LA (ANR09-STRA-09) ainsi que du programme CASDAR PraiFace. 


\section{RÉFÉRENCES BIBLIOGRAPHIQUES}

Albrecht H. (2005). Development of arable weed seedbanks during the 6 years after the change from conventional to organic farming. Weed Research, ${ }^{\circ} 45$, p. 339-350.

Aubry C., Paillat J.-M., Guerrin F. (2006). A conceptual representation of animal wastemanagement at the farm scale: The case of the Reunion Island. Agricultural Systems, $\mathrm{n}^{\circ} 88$, p. 294-315.

Begon M., Pailleux J.-Y., Joly N., Lemery B., Dedieu B. (2009) Les chemins pour durer en élevages bovin laitier : diversité des logiques d'action sur le long terme en Ségala (Massif Central). In I.d. l'élevage-INRA (dir.), Rencontres Recherches Ruminants, Paris, p. 105-108.

Cialdella N., Dedieu B. (2010). What action logics do family livestock farmers have to maintain their activity over the long term? Integrated Farming System Association.

Coquil X., Blouet A., Fiorelli J.-L., Bazard C., Trommenschlager J.-M. (2009). Conception de systèmes laitiers en agriculture biologique : une entrée agronomique. Productions animales, $\mathrm{n}^{\circ} 22$, p. 221-234.

Coquil X., Fiorelli J.-L., Blouet A., Trommenschlager J.-M., Bazard C., Mignolet C. (2011). Conception de systèmes de polyculture élevage laitiers en agriculture biologique : Synthèse de la démarche pas à pas centrée sur le dispositif expérimental INRA ASTER-Mirecourt. Paris, Rencontres autour des Recherches sur les Ruminants, 6 et 7 décembre.

GouttenoireL., Fiorelli J.-L., Trommenschlager J.M., Coquil X., Cournut S. (2010). Understanding the reproductive performance of a dairy cattle herd by using both analytical and systemic approaches: a case study based on a system experiment. Animal, n 4, p. 827-841.

Herrero M., Thornton P. K., Notenbaert A. M., Wood S., Msangi S., Freeman H. A., Bossio D., Dixon J., Peters M., van de Steeg J., Lynam J., Parthasarathy Rao P., Macmillan S., Gerard B., McDermott J., Seré C., Rosegrant M. (2010). Smart Investments in Sustainable Food Production: Revisiting Mixed Crop-Livestock Systems. Science, $\mathrm{n}^{\circ} 327$, p. 822-825.

Holling C. S. (2001). Understanding the Complexity of Economic, Ecological, and Social Systems. Ecosystems, n 4, p. 390-405.

Lamine C., Bellon S. (2009). Conversion to organic farming: a multidimensional research object at the crossroads of agricultural and social sciences. Agronomy for Sustainable Development, $\mathrm{n}^{\circ} 29$, p. 97-112.

Levrouw F., Morales H., Arbeletche P., Malaquin I., Tourrand J.-F., Dedieu B. (2007). Estrategias de largo plazo de los ganaderos uruguayos en situaciones de incertidumbre. Agrociencia, n 11, p. 87-93.

Lotter D. W. (2003). Organic agriculture. Journal of Sustainable Agriculture, $\mathrm{n}^{\circ} 21$, p. 59-128. DOI: 10.1300/J064v21n04_06.

Nousiainen J., Tuori M., Turtola E., Huhtanen P. (2011). Dairy farm nutrient management model. 1. Model description and validation. Agricultural Systems, ${ }^{\circ} 104$, p. 371-382.

Osty P.-L., Landais E. (1993). Fonctionnement des systèmes d'exploitation postorale. Montpellier, Proceedings of the IV International Rangeland Congress, p. 1137-1146.

Vermersch D. (2007). L'échelle versus la gamme. L'éthique en friche. Paris, Éditions Quæ, coll. «Update Sciences et Techniques », p. 61-68. 